\title{
TOPOLOGY OF HOMOLOGY MANIFOLDS
}

\author{
J. BRYANT, S. FERRY, W. MIO, AND S. WEINBERGER
}

\begin{abstract}
We construct examples of nonresolvable generalized $n$-manifolds, $n \geq 6$, with arbitrary resolution obstruction, homotopy equivalent to any simply connected, closed $n$-manifold. We further investigate the structure of generalized manifolds and present a program for understanding their topology.
\end{abstract}

By a generalized $n$-manifold we will mean a finite-dimensional absolute neighborhood retract $X$ such that $X$ is a homology $n$-manifold; that is, for all $x \in X, H_{i}(X, X-\{x\})=H_{i}\left(\mathbb{R}^{n}, \mathbb{R}^{n}-\{0\}\right)$. Generalized manifolds arise naturally as fixed-point sets of group actions on manifolds, as limits of sequences of manifolds, and as boundaries of negatively curved groups. See [BM, Bo, B, GPW]. Such spaces have most of the homological properties of topological manifolds. In particular, generalized manifolds satisfy Poincaré duality [Bo].

Generalized manifolds also share certain geometric and analytic properties with manifolds. Modern proofs of the topological invariance of rational Pontrjagin classes show that Pontrjagin classes can be defined for generalized manifolds and (even better!) that the symbol of the signature operator can be defined for these spaces. See [CSW]. In light of this, the following question seems natural:

Question 1. Is every generalized manifold $X$ homotopy equivalent to a topological manifold?

By [FP], this is true for compact simply connected homology manifolds in all higher dimensions. We shall see below that this is not true in the nonsimply connected case. To continue in this vein, we can consider a strong version of Question 1 that asserts that, for such an $X$, a manifold $M$ can be chosen coherently for all of its open subsets.

Resolution conjecture (see [C]). For every generalized $n$-manifold $X$ there is an $n$-manifold $M$ and a map $f: M \rightarrow X$ such that for each open $U$ in $X$, $f^{-1}(U) \rightarrow U$ is a (proper) homotopy equivalence.

Quinn [Q2] showed that such a resolution is unique if it exists and reduced the resolution conjecture for $X$ to the calculation of a locally defined invariant $I(X) \in H^{0}(X ; \mathbb{Z})$. For $X$ (and $\left.Y\right)$ connected, this invariant satisfies $I(X) \equiv 1$ $(\bmod 8), I(X)=1$ iff $X$ has a resolution, and $I(X \times Y)=I(X) I(Y)$. He also showed that $I(X)$ is an $s$-cobordism invariant of generalized manifolds and that an affirmative solution to Question 1 would imply the resolution conjecture as well. We will henceforth assume that $X$ is connected so that the obstruction $I(X)$ is an integer.

Resolutions are useful maps for studying the geometry of generalized manifolds. For $n \geq 5$, Edwards [E] has characterized $n$-manifolds topologically as

1991 Mathematics Subject Classification. Primary 57N15, 57P10; Secondary 57P05, 57R647.

S. Ferry and S. Weinberger were partially supported by NSF grants. S. Weinberger was partially supported by a Presidential Young Investigator Fellowship. 
being resolvable generalized manifolds that satisfy the following weak transversality condition:

Disjoint disks property (DDP). $X$ has DDP if for any maps $f, g: D^{2} \rightarrow X$ and $\varepsilon>0$ there are maps $f^{\prime}, g^{\prime}: D^{2} \rightarrow X$ with $d\left(f, f^{\prime}\right)<\varepsilon, d\left(g, g^{\prime}\right)<$ $\varepsilon$, and $f^{\prime}\left(D^{2}\right) \cap g^{\prime}\left(D^{2}\right)=\varnothing$. The resolution conjecture would then imply a characterization of topological manifolds as generalized manifolds satisfying DDP.

Our first theorem says that the resolution conjecture is false.

Theorem 1. Generalized manifolds $X$ with arbitrary index $I(X) \equiv 1(\bmod 8)$ exist within the homotopy type of any simply connected closed $n$-manifold, $n \geq 6$.

Corollary 1. There are generalized manifolds that are not homotopy equivalent to manifolds.

The remainder of this announcement is devoted to more positive statements. Some are theorems and some are conjectures.

The first statement requires a little preparation. Recall that an $s$-cobordism is a manifold with two boundary components, each of which includes a simple homotopy equivalence. A basic result in high-dimensional topology asserts that $s$-cobordisms are products.

Theorem 2. Let $\mathscr{S}(M)$ denote the set of s-cobordism classes of generalized manifolds mapping to $M$ by homotopy equivalences which are homeomorphisms on the boundary. If $\operatorname{dim} M \geq 6$, then $\mathscr{S}(M)=\mathscr{S}\left(M \times D^{4}\right)$.

This periodicity theorem was proven by Siebenmann [KS] for manifold structure sets, but there was a $\mathbb{Z}$ obstruction to its universal validity (see $[N]$ ). As suggested by Cappell, the theorem is valid in the class of generalized manifolds. The following theorem will not be precisely stated here but explains the functorial significance of these spaces:

Theorem 3. The algebraic surgery exact sequence (see $[R])$ is valid for highdimensional generalized manifolds up to s-cobordism.

Thus, one knows that if $X$ is simply connected, $\mathscr{S}(X)$ contains generalized manifolds of every index and there is a one-to-one correspondence between $s$-cobordism classes of generalized manifolds homotopy equivalent to $X$ with different indices. Simply connected surgery theory describes the structure of the set of such generalized manifolds with $I(X)=1$.

On the other hand, for "rigid" manifolds $X$ like tori or nonpositively curved manifolds, this theorem asserts that all generalized manifolds homotopy equivalent to $\mathrm{X}$ are $s$-cobordant to the standard model. (This can be proven more directly by using [FJ] and Quinn's resolution theorem [Q1, Q2]).

Our main conjectures are the following:

Homogeneity conjecture. If $X$ is a connected generalized $n$-manifold with DDP, $n \geq 5$, then given $p, q \in X$, there is a homeomorphism $h: X \rightarrow X$ with $h(p)=q$.

$S$-cobordism conjecture. $S$-cobordisms of DDP generalized manifolds are products. 
A corollary of these conjectures is that $n$-dimensional generalized manifolds of a given index that satisfy the DDP are "noncartesian manifolds" modeled on unique local models. The following is very attractive and seems to be a step en route to the $s$-cobordism conjecture.

Revised resolution conjecture. Every generalized manifold $X$ has a resolution by a DDP generalized manifold. If $X$ satisfies DDP, then any such resolution of $X$ is a uniform limit of homeomorphisms.

With these ideas in place, we suggest a modification to another standard conjecture:

Rigidity conjecture. If $\mathrm{X}$ is an aspherical Poincaré complex, then $X$ is homotopy equivalent to a unique generalized manifold satisfying DDP.

If the polyhedron $X$ is nonpositively curved in the sense of [Gr], then one can show, by using [FW], that the resolution obstruction is a well-defined invariant of $\pi_{1}(X)$. Farrell has informed the authors that this also follows from the work of $\mathrm{Hu}$. The Borel rigidity conjecture (which for nonpositively curved $\pi_{1}(X)$ also has been claimed by $\mathrm{Hu}$ ) for manifolds with boundary and the authors' realization theorem imply that a generalized manifold homotopy equivalent to $X$ exists. The $s$-cobordism conjecture would then provide the uniqueness. The authors' methods show that metric analogues of the usual rigidity conjecture are false in situations where this version is correct (up to $s$-cobordism).

Construction of a counterexample among groups of nonpositive curvature could be very useful in terms of producing natural examples of the anticipated local models: these would arise as the spaces at infinity in natural compactifications of the group (see [Gr]).

Finally, we close with the following problem:

Dimension conjecture. The local index described above is realized in dimension four, but no three-dimensional examples of this sort exist.

Remarks on the proof. The spaces are built using controlled surgery as developed in [Q1], [Q2], and [FP]. In surgery one tries to take manifold approximations to a space and make them homotopy equivalent to the target. Our construction is a "controlled" or local version of this. The first step in the construction is to construct a model of a Poincaré space $X$ by gluing together two manifolds with boundary by using a homotopy equivalence between their boundaries.

Successive approximations are refinements of this first step. We construct a model for $X$ with better local Poincaré duality by gluing together two manifold pieces with a homotopy equivalence which is controlled over the first stage. The common boundary is constructed in such a way that its image in the first stage is nearly dense. To produce the required controlled homotopy equivalence, controlled surgery theory comes into the picture. The new generalized manifolds "predicted" by the surgery theory result from the difference between controlled and uncontrolled surgery obstructions on the putative $X$.

Each stage in the construction is a space with better Poincaré duality measured over the previous stage of the construction. As the control on the gluing homotopy equivalences improves, the resulting spaces have better local Poincaré duality. This forces the limit of the approximations to be a homology manifold 
because $X$ is a homology manifold if and only if $X$ satisfies Poincaré duality locally measured over itself, that is, if and only if the constant sheaf is Verdier dual to itself in the derived category of $X$.

Homotopy equivalences with good metric properties are precisely the output of the surgery theory of [FP]. To achieve the desired goal, the codimensionone submanifold we glue along will become denser and denser in $X$. This is inevitable, since the resolution obstruction can be measured on any open subset of a homology manifold $X$. The nonresolvable generalized manifold is obtained as an inverse limit of these approximations. In the limit, all of the approximate self-dualities become a genuine local self-duality.

The surgery theory for generalized manifolds follows from more controlled and relative versions of the basic construction.

\section{REFERENCES}

[B] G. Bredon, Introduction to compact transformation groups, Academic Press, New York, 1972.

[BM] M. Bestvina and G. Mess, The boundary of negatively curved groups, J. Amer. Math. Soc. 4 (1991), 469-481.

[Bo] A. Borel, Seminar on transformation groups, Ann. of Math. Stud., no. 46, Princeton Univ. Press, Princeton, NJ, 1960.

[C] J. Cannon, The characterization of topological manifolds of dimension 5, Proc. Internat. Congr. Math., Helsinki, 1980.

[CSW] S. Cappell, J. Shaneson, and S. Weinberger, Topological characteristic classes for group actions on Witt spaces, C. R. Acad. Sci. Paris 313 (1991), 293-295.

[E] R. D. Edwards, The topology of manifolds and cell-like maps, Proc. Internat. Congr. Math., Helsinki, 1980.

[FJ] F. T. Farrell and L. Jones, Rigidity and other topological aspects of compact nonpositively curved manifolds, Bull. Amer. Math. Soc. (N.S.) 22 (1990), 59-64.

[FP] S. Ferry and E. Pedersen, Epsilon surgery theory, preprint.

[FW] S. Ferry and S. Weinberger, The Novikov conjecture for compactifiable groups, in preparation.

[GPW] K. Grove, P. Petersen, and J. Wu, Geometric finiteness theorems in controlled topology, Invent. Math. 99 (1990), 205-213.

[Gr] M. Gromov, Hyperbolic groups, Essays in Group Theory (S. M. Gersten, ed.), Math. Sci. Res. Inst. Publ., vol. 8, Springer-Verlag, New York, 1987, pp. 75-263.

[KS] R. Kirby and L. C. Siebenmann, Foundational essays on topological manifolds, smoothings, and triangulations, Princeton Univ. Press, Princeton, NJ, 1977.

[N] A. Nicas, Induction theorems for groups of manifold structure sets, Mem. Amer. Math. Soc. vol. 267, Amer. Math. Soc., Providence, RI, 1982.

[Q1] F. Quinn, Resolutions of homology manifolds and the topological characterization of manifolds, Invent. Math. 72 (1987), 267-284.

[Q2] An obstruction to the resolution of homology manifolds, Michigan Math. J. 301 (1987), 285-292.

[R] A. A. Ranicki, The total surgery obstruction, Lecture Notes in Math., vol 763, SpringerVerlag, Berlin and New York, 1979, pp. 275-316. 
(J. Bryant and W. Mio) Department of Mathematics, Florida State University, TallaHASSEE, FLORIDA 32306

E-mail address: bryant@math.fsu.edu

E-mail address: mio@math.fsu.edu

(S. Ferty) Department of Mathematics, State University of New York at Binghamton, Binghamton, New YORK 13901

E-mail address: steve@math.binghamton.edu

(S. Weinberger) Department of Mathematics, University of Chicago, Chicago, Illinois 60637 